\title{
Frictional Sliding without Geometrical Reflection Symmetry
}

\author{
Michael Aldam, ${ }^{1}$ Yohai Bar-Sinai, ${ }^{1}$ Ilya Svetlizky, ${ }^{2}$ Efim A. Brener, ${ }^{3}$ Jay Fineberg, ${ }^{2}$ and Eran Bouchbinder ${ }^{1}$ \\ ${ }^{1}$ Chemical Physics Department, Weizmann Institute of Science, Rehovot 7610001, Israel \\ ${ }^{2}$ Racah Institute of Physics, The Hebrew University of Jerusalem, Givat Ram, Jerusalem 91904, Israel \\ ${ }^{3}$ Peter Grünberg Institut, Forschungszentrum Jülich, D-52425 Jülich, Germany \\ (Received 17 May 2016; revised manuscript received 7 August 2016; published 28 October 2016)
}

\begin{abstract}
The dynamics of frictional interfaces plays an important role in many physical systems spanning a broad range of scales. It is well known that frictional interfaces separating two dissimilar materials couple interfacial slip and normal stress variations, a coupling that has major implications on their stability, failure mechanism, and rupture directionality. In contrast, it is traditionally assumed that interfaces separating identical materials do not feature such a coupling because of symmetry considerations. We show, combining theory and experiments, that interfaces that separate bodies made of macroscopically identical materials but lack geometrical reflection symmetry generically feature such a coupling. We discuss two applications of this novel feature. First, we show that it accounts for a distinct, and previously unexplained, experimentally observed weakening effect in frictional cracks. Second, we demonstrate that it can destabilize frictional sliding, which is otherwise stable. The emerging framework is expected to find applications in a broad range of systems.
\end{abstract}

DOI: 10.1103/PhysRevX.6.041023

Subject Areas: Materials Science, Mechanics

\section{INTRODUCTION}

Understanding frictional sliding is a long-standing challenge with important practical and theoretical implications. It is relevant in diverse physical systems spanning a broad range of scales, from the nanoscale to the planetary scale. A complete analytic treatment of sliding frictional interfaces is generally a formidable task. Two major factors are responsible for the complexity of the problem. First, the friction law, i.e., the constitutive relation that describes the shear traction at the frictional interface, poses experimental challenges and depends on the slip rate and slip history in a highly nonlinear fashion [1-10]. The second factor is the elastodynamics of the sliding bodies, i.e., the timedependent long-range stress transfer mechanisms between different points along the interface. It is particularly challenging when the two bodies are made of different materials and in the generic case in which spontaneously generated interfacial rupture fronts dynamically propagate along the interface [11-23].

A significant simplification in relation to the second factor is obtained when the system possesses reflection symmetry across the interface, i.e., when the two materials are identical, the geometry is symmetric, and the loading configuration is antisymmetric. (Here and elsewhere, we consider macroscopic geometry. Differences in small-scale roughness typically exist and are effectively incorporated

Published by the American Physical Society under the terms of the Creative Commons Attribution 3.0 License. Further distribution of this work must maintain attribution to the author(s) and the published article's title, journal citation, and DOI. into the interfacial constitutive relation.) A prototypical example of such a situation is that of two semi-infinite halfspaces made of identical elastic materials, a situation that was extensively studied in the literature (see, for example, Refs. [24-36]). The main simplification comes from the fact that such a symmetry precludes a coupling between tangential slip and variations in the normal stress. The lack of such symmetry has important implications on the stability of sliding $[11,15,16,20,21,23,37,38]$, the failure mechanism and rupture directionality [17,21,23,37-45]. Physically, this happens because sliding can enhance (reduce) the normal stress, which in turn can inhibit (facilitate) frictional sliding.

The origin of the absence of reflection symmetry is traditionally assumed to be constitutive in nature; i.e., sliding of dissimilar materials is usually considered. This is known as the bimaterial effect. Sliding along such bimaterial interfaces has been quite extensively studied in the literature, and this material contrast is thought to have important implications for frictional dynamics [11,1517,20-23,37-45]. The purpose of this paper is to explore the possibility of asymmetry of a geometric origin, i.e., sliding of two bodies made of the same material without geometrical reflection symmetry. Examples of such geometries are depicted in Fig. 1: sliding of two blocks with different thickness in the direction orthogonal to sliding, an experimental setup that was used in various recent works [46-50] and will be theoretically addressed below [panel (a)]; sliding of a block of finite height $H$ over a semiinfinite bulk, a simple example to be analyzed in depth in this work [panel (b)]; finally, an idealized sketch of tectonic subduction motion [panel (c)], a situation in which one 


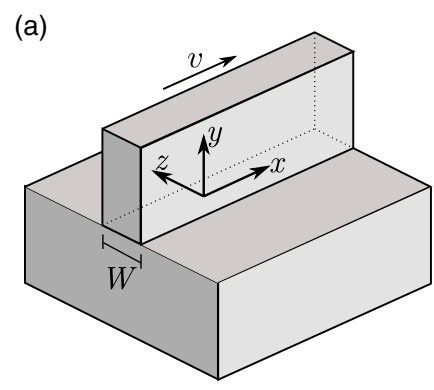

(b) $\sigma_{y y}=-\sigma_{0}$

(c)

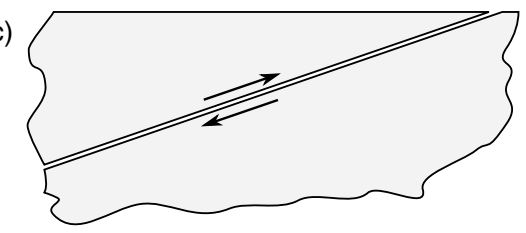

FIG. 1. Examples of physical systems featuring frictional interfaces separating bodies made of identical materials without geometrical reflection symmetry. (a) A thin block sliding over a thicker block. (b) A block of finite height $H$ sliding atop a semiinfinite bulk. Sliding occurs in the $x$ direction. (c) An idealized schematic geometry of tectonic subduction motion.

lithospheric plate is subducted beneath another one and is responsible for most of the large magnitude earthquakes ("megathrust") occurring on the Earth's crust (see Refs. [51-56], for example). Obviously, many other sliding geometries that lack reflection symmetry can be conceived. Generally speaking, this situation is expected to be the rule rather than the exception since no physical system features perfect reflection symmetry.

In this paper, we lay out a rather general theoretical framework to address frictional sliding in the absence of geometrical reflection symmetry and support it by extensive experiments. A major outcome is that the effect of geometric asymmetry resembles, sometimes qualitatively and sometimes semiquantitatively, that of material asymmetry. Consequently, many results obtained for bimaterial interfaces are also relevant to interfaces separating bodies made of identical materials with different geometries. As first applications, two main results are obtained within the newly developed framework:

(i) A novel explanation of a sizable weakening effect observed in recent experiments on rupture-fronts propagation along frictional interfaces [49]. The weakening effect is directly linked to geometric asymmetry and is shown experimentally to disappear in its absence. This result has important implications for the failure dynamics of frictional interfaces.

(ii) We demonstrate that geometric asymmetry can destabilize frictional sliding that is otherwise stable. We consequently expect geometric asymmetry to play an important role in frictional instabilities.

The emerging framework should find additional applications in a broad range of frictional systems.

\section{GENERAL FRAMEWORK}

Consider two blocks in frictional contact. At this stage, the discussion remains completely general, allowing the two blocks to be made of different materials, to feature different geometries, and to experience general external loadings. We denote the displacement vector fields in the two blocks as $\boldsymbol{u}^{(1)}(\boldsymbol{x}, t)$ and $\boldsymbol{u}^{(2)}(\boldsymbol{x}, t)$, where the superscripts correspond to the upper and lower blocks, respectively. Each of these satisfies the momentum balance equation $\nabla \cdot \boldsymbol{\sigma}=\rho \ddot{\boldsymbol{u}}$, where $\rho$ is the mass density of each block. Cauchy's stress tensor $\sigma$ is related to the displacement gradient tensor $\nabla \boldsymbol{u}$ according to the isotropic Hooke's law $(1+\nu) \mu\left[\nabla \boldsymbol{u}+(\nabla \boldsymbol{u})^{\mathrm{T}}\right]=\boldsymbol{\sigma}-\nu(\boldsymbol{I} \operatorname{tr} \boldsymbol{\sigma}-\boldsymbol{\sigma})$. Here, $\boldsymbol{I}$ is the identity tensor, $\nu$ is Poisson's ratio, and $\mu$ is the shear modulus of each block. The coordinates are chosen such that the interface lies along the $x$ axis, which is also the direction of sliding (see Fig. 1). The direction normal to the interface is the $y$ axis, and the interface is the surface $y=0$. The $z$ axis is in the thickness direction, where $z=0$ is the center line. While the formulation below and the analysis in Sec. IV are two dimensional (2D), in Sec. III we see that three-dimensional (3D) effects involving the $z$ coordinate play an important role.

Since the bulk equations are linear, one can separately analyze each interfacial Fourier mode, i.e., write $\boldsymbol{u}^{(n)}(x, y=0, t)=\boldsymbol{u}^{(n)} e^{i k(x-c t)}$, where $k>0, c$ is the complex propagation (phase) velocity and $n=1,2$. The relation between the interfacial displacements and stresses is also linear and can be written as $u_{i}^{(n)}=M_{i j}^{(n)}(c, k) \sigma_{y j}^{(n)}$, where the matrix $\boldsymbol{M}^{(n)}$ can be obtained from the Green's function of the corresponding medium and $\sigma_{y i}^{(n)}$ are the interfacial stresses, i.e., at $y=0$. For example, under quasistatic conditions for semi-infinite blocks in $2 \mathrm{D}$, this relation for the lower block (i.e., the block at $y<0$ ) takes the form [57]

$$
\left(\begin{array}{c}
u_{x} \\
u_{y}
\end{array}\right)=\frac{1}{\mu k}\left(\begin{array}{cc}
1-\nu & -\frac{i}{2}(1-2 \nu) \\
\frac{i}{2}(1-2 \nu) & 1-\nu
\end{array}\right)\left(\begin{array}{c}
\sigma_{x y} \\
\sigma_{y y}
\end{array}\right) .
$$

The essence of frictional motion is that the displacement field is discontinuous across the interface. We denote the slip discontinuity at the frictional interface by

$$
\epsilon_{i}(x) \equiv u_{i}^{(1)}\left(x, y=0^{+}\right)-u_{i}^{(2)}\left(x, y=0^{-}\right) .
$$

On the interface, $y=0$, no separation or interpenetration between the bulks implies $\epsilon_{y}=0$ and continuity of $\sigma_{y i}$. Together with the known dynamic response matrices $\boldsymbol{M}^{(n)}$, these requirements can be used to calculate the relation between the slip discontinuity and the interfacial stresses of the composite system that consists of both bulks. Following Ref. [58], this calculation is done by noting that for $y=0$, 
we have $\sigma_{y i}=\left(\boldsymbol{M}^{(1)}-\boldsymbol{M}^{(2)}\right)_{i j}^{-1} \epsilon_{j}$. Thus, the response of the composite system in 2D reads (in Fourier space)

$$
\begin{gathered}
\sigma_{x y}=\mu^{(1)} k G_{x}(c, k) \epsilon_{x}(k), \\
\sigma_{y y}=i \mu^{(1)} k G_{y}(c, k) \epsilon_{x}(k),
\end{gathered}
$$

where we defined the elastic response functions $G_{x}(c, k) \equiv\left(\mu^{(1)} k\right)^{-1}\left(\boldsymbol{M}^{(1)}-\boldsymbol{M}^{(2)}\right)_{x x}^{-1} \quad$ and $\quad G_{y}(c, k) \equiv$ $-i\left(\mu^{(1)} k\right)^{-1}\left(\boldsymbol{M}^{(1)}-\boldsymbol{M}^{(2)}\right)_{y x}^{-1}$. In other words, the $G_{i}$ 's can be expressed as functions of the response coefficients of both bulks. Note that the imaginary unit $i$ is included in Eq. (3) for convenience. Note also that we use the same notation for a function and its Fourier transform, as they are easily distinguishable by the context or the stated arguments (e.g., $k$ or $x$ ).

The central player in the analysis to follow is $G_{y}$, which represents the elastodynamic coupling between tangential slip and normal traction along the interface. In systems with complete reflection symmetry along $y=0$, this coupling is precluded by symmetry. To see this, note that, in this case, the off-diagonal elements of $\boldsymbol{M}^{(1)}$ and $\boldsymbol{M}^{(2)}$ are identical [57], and thus $\boldsymbol{M}^{(1)}-\boldsymbol{M}^{(2)}$, as well as its inverse, is diagonal. This result immediately implies $G_{y}=0$. In what follows, we study two important frictional problems in which the sliding bodies are made of identical materials, i.e., $\rho^{(1)}=\rho^{(2)} \equiv \rho, \mu^{(1)}=\mu^{(2)} \equiv \mu$, and $\nu^{(1)}=\nu^{(2)} \equiv \nu$, yet reflection symmetry relative to the interface is absent because of asymmetry in the geometry of the bodies, leading to $G_{y} \neq 0$. These problems highlight the importance of geometrical asymmetry to frictional sliding and its relation to the conventional bimaterial effect.

\section{THIN-ON-THICK SYSTEMS AND THE PROPAGATION OF FRICTIONAL CRACKS}

The first problem that we examine, which is directly motivated by recent experimental observations [44,49], is depicted in Fig. 1(a). In this system, a thin block of width $W=5.5 \mathrm{~mm}$ is pushed along its length (the $x$ axis) on top of a significantly thicker block (here, $30 \mathrm{~mm}$ ). This thinon-thick experimental setup was used in various studies [46-50], where a transparent glassy polymer [poly (methylmethacrylate), PMMA] was used. The transparent material allows a direct real-time visualization and quantification of a fundamental interfacial quantity: the real contact area $A_{r}$. The latter is the sum over isolated microcontacts formed because of the small-scale roughness of macroscopic surfaces.

$A_{r}$ is typically orders of magnitude smaller than the nominal contact area $A_{n}$. The ratio $A \equiv A_{r} / A_{n} \ll 1$ plays a critical role in interfacial dynamics [2,3,46-49,59-62] because the frictional stress is proportional to $A$, $\sigma_{x y} \propto A$; i.e., the larger the real contact area the larger the frictional resistance. $A$ itself depends on the normal stress and also on the slip history of the interface according to

$$
\sigma_{x y} \propto A \propto \sigma_{y y}(1+\psi),
$$

where $\psi$ is an internal variable characterizing the state of the interface. Frictional sliding leads to reduction of $\psi$, i.e., to a reduction of the contact area $[1-3,6,61,63]$. In the absence of sliding, $\psi$ (and hence $A$ ) grows logarithmically with time, a process known as frictional aging $[1,3,48,64,65]$.

In Ref. [49], it was found that sliding is mediated by a succession of cracklike rupture fronts propagating along the frictional interface and that these fronts are surprisingly well described by the classical theory of shear cracks propagating along an interface separating identical materials, linear elastic fracture mechanics (LEFM). The variation of $A$ along a few of these fronts is shown here in Fig. 2(a). It is seen that the rupture fronts involve a significant overall reduction of the contact area, which weakens the interface (i.e., reduces $\psi$ ) and facilitates sliding. We focus our attention on a distinct feature of these curves: As observed in Fig. 2(a), fronts that travel at $90 \%$ of the Rayleigh wavespeed $c_{R}$, here $c_{R} \simeq 1237 \mathrm{~m} / \mathrm{s}$ (for plane-stress conditions, see Ref. [57]), or slower (not shown), feature a monotonic decrease of $A$. However, in fronts propagating even closer to $c_{R}$, $A$ features a nonmonotonic behavior; i.e., $A$ undershoots the asymptotic value $A_{\infty}$ (i.e., $A$ as $x \rightarrow-\infty$ ) and then rapidly increases, at a rate way too high to be explained by slow frictional aging. This nonmonotonic behavior remained unexplained in Ref. [49], where it was stated that "the nonmonotonic behavior of $A$... suggests interesting dynamics as $c \rightarrow c_{R} \ldots$. "

In Fig. 2(b), we show the spatial profiles of the slip velocity $v$, corresponding to the contact area profiles shown in Fig. 2(a). These profiles were calculated from the experimental data using the simplest cohesive zone model [66-68], which is consistent with the measurements of the fracture energy and cohesive zone size (see Ref. [57] for more details). This model, while generally used to describe identical materials, is motivated by the empirical observation [57] that the strain fields are, to first order, quite similar in the thin-on-thin and thin-on-thick setups. This approximation would, of course, have to be modified in cases of strong material contrast, where the fields on both sides of the interface differ strongly [44].

We denote the maximum slip velocity in these profiles by $v_{m}$. Next, in order to quantify the nonmonotonic effect, we define the magnitude of the undershoot $\Delta A$ as the difference between the asymptotic value $A_{\infty}$ and the minimum of the profile over the range $-5 \mathrm{~mm}<x<0$, which is the typical spatial range for which $\Delta A>0$ is observed in the thin-on-thick setup [see Fig. 2(a)]. In Fig. 2(c), $\Delta A / A_{\infty}$ is plotted vs $v_{m}$ (red symbols), demonstrating that the former is quasilinear (i.e., predominantly 

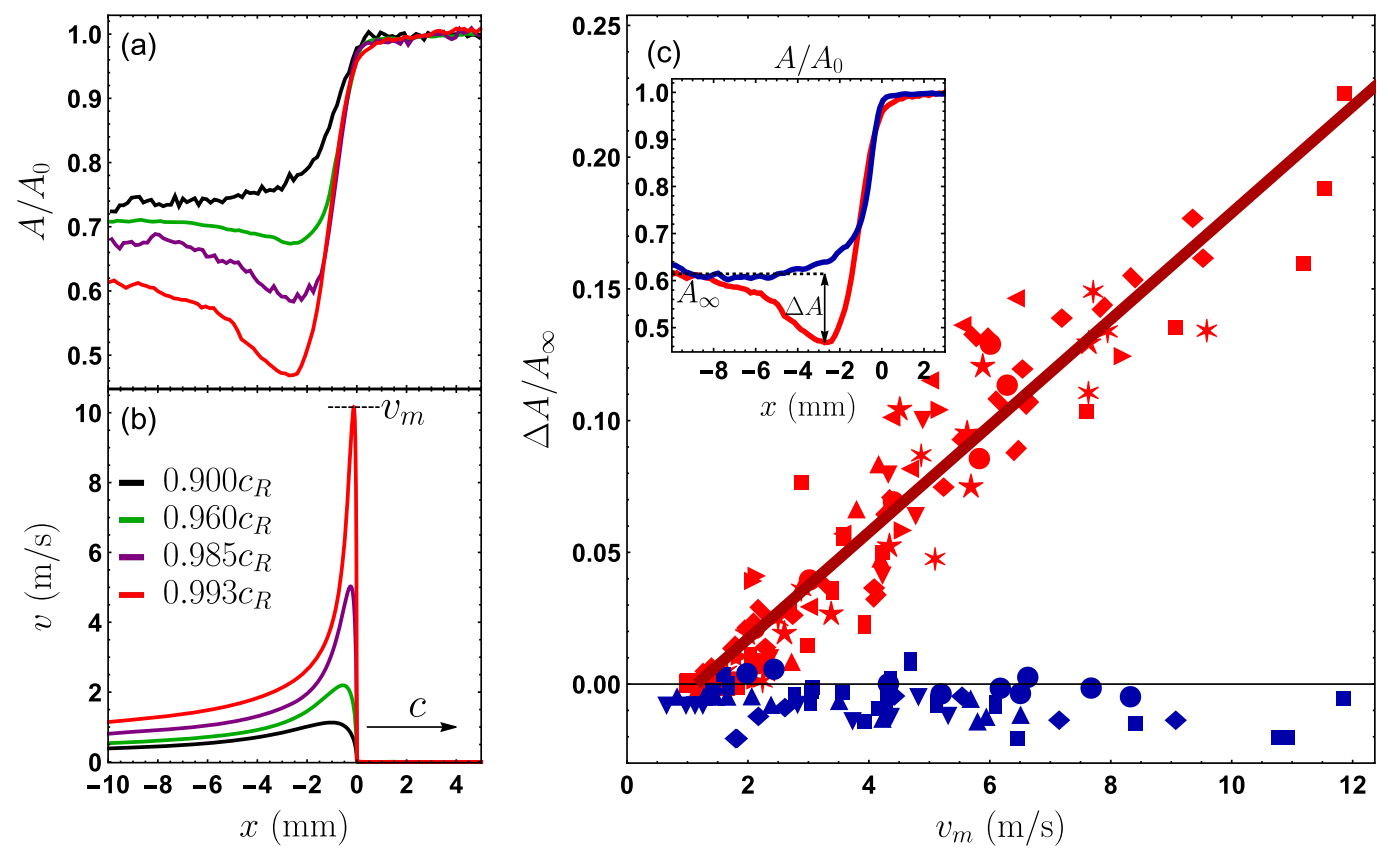

FIG. 2. Experimental results. (a) Snapshots of the spatial profile of the contact area $A$ of rupture fronts in the "thin-on-thick" setup [see Fig. 1(a) and Ref. [49] for additional details]. These fronts propagate to the right at velocities $c$ indicated in the legend of panel (b) $\left(0.900 c_{R}<c<0.993 c_{R}\right.$, see Ref. [57]), where $x=0$ corresponds to the tip of each rupture front. The contact area is normalized by its value $A_{0}$ before the passage of the front. (b) The slip-velocity profiles corresponding to the snapshots in panel (a) (see Ref. [57] for details). (c) $\Delta A / A_{\infty}$, where $\Delta A$ is the magnitude of the real contact area undershoot and $A_{\infty}$ is the asymptotic value (see inset) vs the maximal slip velocity $v_{m}$ [see panel (b)] for both the thin-on-thick setup (red symbols) and the geometrically symmetric "thin-on-thin" setup (blue symbols). Different symbols correspond to different experiments, and their size roughly corresponds to the measurement error. The red line is the best linear fit for the red symbols. (Inset) The contact area profile for $c=0.993 c_{R}$ in the thin-on-thick setup [red line, already appearing in panel (a)] and in the geometrically symmetric thin-on-thin setup (blue line).

linear) in the latter. Note that the spread in the data does not allow us to identify any systematic deviations from linearity. We stress that the effect is not only qualitatively novel, i.e., the existence of a nonmonotonic contact area behavior $\Delta A / A_{\infty}>0$, but it is also quantitatively important. As Fig. 2(c) shows, the local reduction in the real contact area $\Delta A / A_{\infty}$ can reach nearly $25 \%$. This is a large quantitative effect, compared to other documented frictional effects, implying the existence of significant local frictional weakening, which can significantly influence interfacial dynamics.

What is the source of this nonmonotonicity, why does it scale quasilinearly with the slip velocity, and why does it appear only at sonic propagation velocities? Such behavior has recently been observed in Ref. [44] when investigating the frictional motion of bimaterial interfaces in a geometrically symmetric system. There, a very large local reduction of $A$ was observed at sonic propagation velocities, but it entirely disappeared when the upper and lower blocks were made of the same material. We propose that the same thing happens in our case, only here it is due to geometric asymmetry. In other words, we suggest that the nonmonotonicity of $A$ stems from the absence of geometrical reflection symmetry of the two blocks, i.e., from the difference in their thickness. If this is true, then the fast nonmonotonic variation of $A$ is not an intrinsically frictional phenomenon, i.e., a result of the dynamics of the state of the interface $\psi$, but rather an elastodynamic effect emerging from the coupling between slip and normal stress variations, solely induced by geometrical effects. In terms of Eq. (4), we propose that $\psi$ is monotonic and that the nonmonotonicity of $A$ results from a nonmonotonic behavior of $\sigma_{y y}$.

Our strategy in testing and exploring this idea is twofold. First, our idea can be directly tested by a definitive experiment. In other words, we expect that when the width of the lower block equals that of the upper one, i.e., in a thin-on-thin setup, the nonmonotonicity in $A$ disappears altogether even in the limit $c \rightarrow c_{R}$. We performed this experiment, as in Ref. [44], and we present a representative example (for $c=0.993 c_{R}$ ) in the inset of Fig. 2(c) (blue line). The curve is indeed monotonic. Moreover, note that the asymptotic value $A_{\infty}$ is the same as that in the thin-onthick setup [cf. Fig. 1(a), for the same propagation velocity], even though the latter exhibits a large undershoot. In Fig. 2(c) (main panel), we added $\Delta A / A_{\infty}$ of many rupture fronts in the thin-on-thin setup (blue symbols). Note that $\Delta A / A_{\infty}$ is indeed very close to zero (small negative values simply correspond to monotonic behavior); i.e., all of the $A$ profiles in the thin-on-thin setup are 
monotonic. This direct experimental evidence provides unquestionable support of our basic idea that the nonmonotonic behavior corresponding to the thin-on-thick setup data in Fig. 2(a) emerges from the absence of geometrical reflection symmetry.

Next, our aim is to develop a theoretical understanding of the origin of nonmonotonicity. The challenge is to explain both the fact that it emerges at asymptotic propagation velocities $\left(c \rightarrow c_{R}\right)$ and the quasilinear relation between $\Delta A / A_{\infty}$ and $v_{m}$. The complete problem, involving a thin block sliding atop a thicker one, is a very complicated 3D elastodynamic problem. We approach the problem by breaking it into two steps. First, we perform a simplified analysis, invoking physically motivated approximations, which allow us to reduce the mathematical complexity of the problem and gain analytic insight into it. The major simplification is to consider the corresponding quasistatic problem instead of the full elastodynamic one. The physical rationale for this is clear: The absence of geometrical reflection symmetry should also manifest its generic implications in the framework of static elasticity, and hence the simplified analysis is expected to reveal the origin of the nonmonotonicity of the real contact area. Then, in the second step, we use the static results in an effective dynamic calculation, to be explained below.

The main outcome of the first step is that the static 3D problem can be approximately mapped onto a $2 \mathrm{D}$ problem involving two elastically dissimilar materials. In other words, we show that the geometric asymmetry can be approximately mapped onto an effective constitutive asymmetry, i.e., an effective material contrast. To see how this

$$
\boldsymbol{M}^{\mathrm{eff}} \simeq \frac{1}{\mu k}\left(\begin{array}{c}
(1-\nu) B(q) \\
\frac{i}{2}(1-2 \nu)\left(1-e^{-(|q| / 2)}\right)
\end{array}\right.
$$$$
\left.\begin{array}{c}
-\frac{i}{2}(1-2 \nu)\left(1-e^{-(|q| / 2)}\right) \\
(1-\nu) B(q)
\end{array}\right)
$$

ese assume that both blocks are infinite in the $y$ direction and that the thicker (lower) block is also infinite in the $z$ direction. In other words, the lower block is replaced by a semi-infinite $3 \mathrm{D}$ half-space, which allows us to use the well-known interfacial Green's function [69]. More specifically, the 3D real-space Green's function matrix $\hat{\boldsymbol{M}}^{3 \mathrm{D}}\left(\boldsymbol{r}-\boldsymbol{r}^{\prime}\right)$ [69] allows us to express the interfacial displacements at a point $\boldsymbol{r}=(x, y=0, z=0)$ on the symmetry line, $\boldsymbol{u}(\boldsymbol{r})=\left(u_{x}, u_{y}\right)$, induced by a point force applied by the upper block at $\boldsymbol{r}^{\prime}=\left(x^{\prime}, y=0, z^{\prime}\right)$, $\boldsymbol{F}\left(\boldsymbol{r}^{\prime}\right)=\left(F_{x}, F_{y}\right)$. We assume that the latter does not contain an out-of-plane component, i.e., $F_{z}=0$, which, in principle, could emerge from frustrated Poisson expansion at the interface. It is reasonable, though, to neglect it to leading order.

We physically expect shear tractions to be uniform across the thickness $W$; hence, they are taken to be constant for $|z| \leq(W / 2)$ (and, of course, to vanish for $|z|>(W / 2)$ ). Thus, we obtain

$$
\left(\begin{array}{l}
u_{x} \\
u_{y}
\end{array}\right)=\boldsymbol{M}^{\mathrm{eff}}(k)\left(\begin{array}{c}
\sigma_{x y} \\
\sigma_{y y}
\end{array}\right)
$$

where the effective 2D response matrix $\boldsymbol{M}^{\mathrm{eff}}(k)$ of the thicker (lower) block is given by the Fourier transform of $\hat{\boldsymbol{M}}^{3 \mathrm{D}}$ over the strip $|z| \leq(W / 2)$,

$$
\boldsymbol{M}^{\mathrm{eff}}(k)=\int_{-\infty}^{\infty} d x^{\prime} \int_{-\frac{W}{2}}^{\frac{W}{2}} d z^{\prime} e^{i k\left(x-x^{\prime}\right)} \hat{\boldsymbol{M}}^{3 \mathrm{D}}\left(x-x^{\prime}, z^{\prime}\right) .
$$

The integration can be carried out, resulting in

where $q \equiv k W$ and $B(q)=\pi^{-1} \int_{0}^{q} \mathcal{K}_{0}\left(q^{\prime} / 2\right) d q^{\prime}\left[\mathcal{K}_{0}(z)\right.$ is the modified Bessel function of the second kind of order 0$]$. The outcome of the analysis, which is presented in full detail in Ref. [57], is that $\boldsymbol{M}^{\text {eff }}(k)$ appears to identify with the $2 \mathrm{D}$ response matrix of Eq. (1), if one defines the effective elastic moduli of the lower (thicker) block as

$$
\begin{aligned}
\mu^{\mathrm{eff}}(q) & \simeq \frac{\mu}{2(1-\nu) B(q)-(1-2 \nu)\left(1-e^{-(|q| / 2)}\right)}, \\
\nu^{\mathrm{eff}}(q) & \simeq \frac{(1-\nu) B(q)-(1-2 \nu)\left(1-e^{-(q \mid / 2)}\right)}{2(1-\nu) B(q)-(1-2 \nu)\left(1-e^{-(|q| / 2)}\right)} .
\end{aligned}
$$

These are plotted in Fig. 3(a).

The mapping of the $3 \mathrm{D}$ problem onto an effective $2 \mathrm{D}$ problem is formally valid as long as the interfacial stresses (and hence displacements) in Eq. (5) are approximately localized in Fourier $k$ space. Otherwise, Eq. (5) will not identify with Eq. (1) because of the extra $k$ dependence of $\mu^{\text {eff }}(k W)$ and $\nu^{\text {eff }}(k W)$, which is a result of the 3D nature of the original problem. We note in passing that in the limit $q=k W \gg 1, \mu^{\text {eff }} \rightarrow \mu$ and $\nu^{\text {eff }} \rightarrow \nu$, which corresponds to 2D plane-strain conditions [71]. This result is expected for small wavelengths, for which the thinner block also appears infinitely thick, and hence, it is a consistency check on our calculation. The important observation, though, as is clearly seen in Fig. 3(a), is that for the thicker block $\mu^{\text {eff }}(k)>\mu$ for all experimentally relevant $k$ 's [72]. This suggests that the thicker block is effectively stiffer than the thinner one, as hypothesized in Refs. [73,74], where the thicker block was assumed to correspond to plane-strain conditions in numerical simulations. In other words, the main physical insight gained from the performed analysis is 

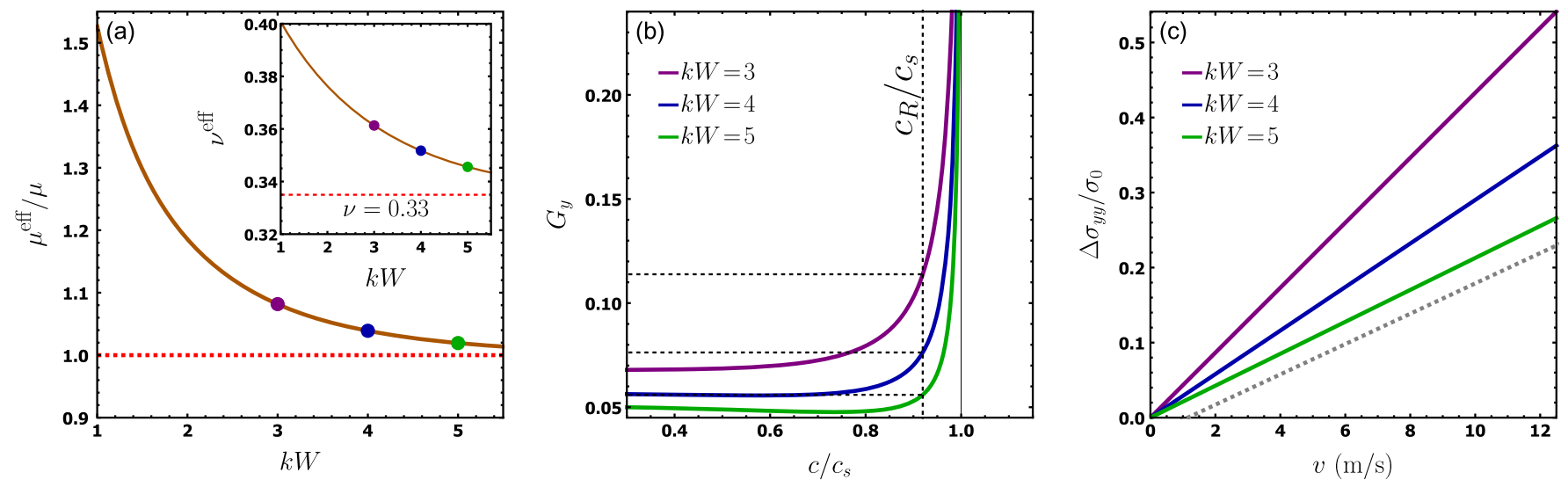

FIG. 3. Analytical results. (a) The effective shear modulus $\mu^{\text {eff }}$ of the thicker block, in units of $\mu$, vs the dimensionless wave number $q=k W$, cf. Eq. (7). (Inset) The variation of the effective Poisson's ratio $\nu^{\text {eff }}(q)$ with $q=k W$. In both the main panel and the inset we used $\nu=0.33$, which is relevant for PMMA [49,70]. (b) The response function $G_{y}$, quantifying the effective bimaterial contrast according to $\mu^{\mathrm{eff}}(q)$ and $\nu^{\mathrm{eff}}(q)$ (for the thicker block; the thinner one is represented by plane-stress conditions), corresponding to selected values of $q=k W$. The corresponding values of the elastic moduli $\mu^{\text {eff }}(q)>\mu$ and $\nu^{\text {eff }}(q)>\nu$ are marked in panel (a) and its inset using the same color code. (c) $\Delta \sigma_{y y}$ given in Eq. (8), normalized by the experimentally applied normal stress $\sigma_{0}=4.5 \mathrm{MPa}$, vs the slip velocity $v$, where the propagation velocity was set to $c=c_{R} \simeq 1237 \mathrm{~m} / \mathrm{s}$. The gray dashed line is the red line in Fig. 2 (c).

that geometric asymmetry gives rise to an effective material contrast.

With this physical insight in hand, we now aim at addressing the nonmonotonicity of $A$ discussed in panels (a) and (c) of Fig. 2. The 3D static analysis presented above may not yield quantitatively accurate predictions when strongly elastodynamic 2D interfacial rupture fronts are considered. Yet, we believe that the insight embodied in the relations $\mu^{\text {eff }}(k)>\mu$ and $\nu^{\text {eff }}(k)>\nu$ is physically robust; hence, we try to explore their quantitative implications in relation to the experimental observations in the dynamic regime.

To accomplish this, we consider the 2D dynamic transfer function $G_{y}(c, k)$ in Eq. (3) and assume that it approximately describes the experimental system when the effective moduli $\mu^{\text {eff }}(k)>\mu$ and $\nu^{\text {eff }}(k)>\nu$ are used for the thicker (lower) block and when plane-stress conditions [71] are assumed for the thinner (upper) block. Note that it is justified to treat the heights of the two blocks as infinite since the experimental rupture fronts are so fast that they do not interact with the upper and lower boundaries before traversing the whole system. Therefore, Eq. (3) can be rewritten as

$$
\Delta \sigma_{y y}(c, k, v)=-c^{-1} \mu G_{y}\left[c, k ; \mu^{\mathrm{eff}}(k), \nu^{\mathrm{eff}}(k)\right] v,
$$

where we used $v=\dot{\epsilon}_{x}=-i c k \epsilon_{x}$ for a constant propagation velocity $c$.

The 2D infinite-system dynamic transfer function $G_{y}(\cdot)$ in Eq. (8) was calculated quite some time ago by Weertman for the sliding of dissimilar materials [12]. We reiterate that the basic idea here is to use a known result for dissimilar materials to represent a system composed of identical materials with geometric asymmetry, utilizing the effective moduli derived in Eq. (7), $\mu^{\text {eff }}(k)>\mu$ and $\nu^{\text {eff }}(k)>\nu$. In the presence of any contrast between the shear moduli of the materials, $G_{y}(c)$ is finite, and it increases significantly at elastodynamic velocities (in fact, it diverges when $c$ approaches the shear wave-speed $c_{s}$ of the more compliant material), as shown in Fig. 3(b). Thus, we expect rupture fronts that propagate at near-sonic velocities to be accompanied by a significant reduction in the local normal stress as implied by Eq. (8), locally reducing the real contact area. In turn, this reduces the interfacial strength, which facilitates sliding. This result is consistent with the experimental observations of Fig. 2(a), where the nonmonotonicity of $A$ becomes substantial at asymptotic propagation velocities $\left(c \rightarrow c_{R}\right)$. This normal stress reduction is also remarkably similar to the recent observations of Ref. [44] in bimaterial systems, a similarity that further strengthens the analogy between geometric asymmetry and material asymmetry.

The connection between geometric and material asymmetries is yet further strengthened when the directionality of rupture is considered. The sub-Rayleigh $\left(c<c_{R}\right)$ rupture fronts, shown in Fig. 2(b), propagate from left to right, in the direction of sliding of the thinner (upper) block [see also Fig. 1(a)]. Sub-Rayleigh rupture fronts that are accompanied by normal stress reduction are known to propagate in the direction of sliding of the more compliant material in a bimaterial setup, the so-called "preferred direction" $[12,17,44]$. This is fully consistent with our result that the thinner (upper) block is effectively softer than the thicker (lower) block (or alternatively, that the thicker block is effectively stiffer than the thinner one).

The quasilinearity of $\Delta A$ with the (maximal) slip velocity, observed in Fig. 2(c), naturally emerges from 
Eq. (8). To see this, note that $\Delta A \propto \Delta \sigma_{y y}$ according to Eq. (4) (recall that $\psi$ in that equation is expected to be monotonic) and that $c$ remains close to $c_{R}$ to within a few percent. In this regime $\left(c \simeq c_{R}\right), G_{y}$ does not change appreciably as a function of $c$, while the maximal $v$ varies quite substantially [cf. Fig. 2(a)]. This means that while $c \simeq c_{R}$ is required for the existence of the weakening effect, its variability is mainly determined by $v$. Putting this together, we obtain $\Delta A \propto v$. To obtain some estimate of the proportionality factor between $\Delta A$ and $v$ along this line of reasoning, we interpret $\Delta \sigma_{y y}$ in Eq. (8) to be a function of $v$ alone, with $c=c_{R}$ and $k \sim \mathcal{O}\left(W^{-1}\right)$, where $\mu^{\text {eff }}(k)>\mu$ [cf. Fig. 3(a)].

The results for $\Delta \sigma_{y y}(v)$ with $k W=3,4,5$, normalized by the experimentally applied normal stress $\sigma_{0}$, are shown in Fig. 3(c). The slope of the $k W=5$ line is very close to the slope of the linear fit in Fig. 2(c), which was added to Fig. 3(c) for comparison (gray dashed line). Note that the experimental line features a finite $v$ intercept, which is absent in the theoretical one. This is expected since the undershoot $\Delta A$ is generally susceptible to variations of both $\sigma_{y y}$ and the fracture of contacts [variations of $\psi$ in Eq. (4)]. For low values of $v$, variations of $\sigma_{y y}$ should be small, and the spatial profile of $A$ is therefore dominated by variations of $\psi$. Therefore, $A(x)$ should be monotonic in space, similar to the spatial profile in the thin-on-thin setup, thus rendering any undershoots (i.e., $\Delta A$ ) unmeasurable.

This quantitative agreement should be taken with some caution in light of the various approximations invoked above. Yet, the existence of a characteristic wave number $k W=5$ is not unreasonable, as the typical scale of the velocity peaks [see Fig. 2(b)], the spatial scale of the undershoot in the contact area [see Fig. 2(a)], and $W$ are all in the $\mathrm{mm}$ scale. Furthermore, the relative magnitudes of the slopes in Fig. 3(c) provide a testable prediction for how the slope decreases with increasing $W$. This should be experimentally tested in the future. Finally, as $W$ increases and approaches the width of the lower block, the nonmonotonicity is predicted to disappear, as demonstrated experimentally in Fig. 2(c) (blue symbols).

The results presented in this section demonstrate that global geometric features of the sliding bodies in a frictional problem (here, a difference in their thickness) affect the frictional resistance to sliding and actually make it easier for interfacial rupture fronts that mediate sliding to propagate. In fact, the effect of geometric asymmetry is maximal at the extreme rupture velocities that are the norm in frictional sliding. This reduction in frictional dissipation applies to any engineering or tribological system involving identical materials and geometric asymmetry. As such, it implies that the design and friction control of any real-life tribological application must take into account not only the interfacial properties but also the relative size of the sliding bodies. In the next section, we show that the same concept applies to another class of important sliding friction problems, where a different form of geometric asymmetry controls the dynamic response of the system.

\section{STABILITY OF FRICTIONAL SLIDING}

We now focus on a different, yet conceptually related, physical situation in which geometric asymmetry plays a crucial role as well. While in Sec. III geometric asymmetry was associated with a difference in the thickness of the sliding bodies, here its origin is a difference in their height. Moreover, while in Sec. III we addressed the propagation of spatially localized interfacial cracks, here the focus is on the stability of homogeneous sliding. Yet, in both cases, a geometry-induced coupling between interfacial slip and normal stress variations, encapsulated in the function $G_{y}$ in Eq. (3), is the dominant physical player.

We consider an elastic block of height $H^{(1)}$ sliding atop a block of height $H^{(2)}=\eta H^{(1)}$ (with a dimensionless positive $\eta, 0<\eta<\infty)$, both made of the same material under plane-strain conditions [71], as depicted in Fig. 1(b). Note that $\eta=1$ corresponds to a symmetric system. The blocks initially slide at a fixed velocity, and all of the fields are assumed to reach a steady state. A homogeneous compressive normal stress $\sigma_{y y}^{(1)}=-\sigma_{0}$ is imposed at both $y=H^{(1)}$ and $y=-H^{(2)}$. In addition, a constant velocity $\dot{u}_{x}^{(1)}=v$ in the positive $x$ direction is imposed at $y=H^{(1)}$ and $\dot{u}_{x}^{(2)}=0$ at $y=-H^{(2)}$. In this problem, unlike the problem considered in Sec. III, the interfacial dynamics are coupled to the boundaries at $y=H^{(1)},-H^{(2)}$; hence, the heights $H_{1,2}$ are expected to play a central role here.

To fully define the problem, one needs to specify the frictional boundary condition at the interface. Friction is commonly modeled as a linear relation between the interfacial normal stress and the interfacial shear stress (frictional stress), i.e.,

$$
\sigma_{x y}=-f(\cdot) \sigma_{y y}
$$

where $f(\cdot)$ represents the friction law. Our major goal here is to understand the destabilizing effect associated with geometric asymmetry, i.e., $\eta \neq 1$, which, to the best of our knowledge, has not been studied before. Consequently, in order to isolate the geometric effect, below we focus on situations in which friction is intrinsically stabilizing such that any instability, if it exists, is associated with the absence of geometrical reflection symmetry.

To achieve this, we proceed in two steps. First, in Sec. IVA, we present a simplified analysis involving a simple velocity-dependent friction law and strong geometrical asymmetry. This will allow us to gain much insight into the role of geometric asymmetry in frictional sliding and to clearly identify the physical origin of instability. Then, in Sec. IV B, we present a significantly generalized analysis for a realistic friction law, including an internal state variable and an interfacial memory length, and for any 
level of geometric asymmetry. The emerging results strengthen the findings of Sec. IVA and extend them.

\section{A. Simplified analysis: Velocity-dependent friction and large geometric asymmetry}

As a primer, here we use a simple friction law where $f(\cdot)$ in Eq. (9) depends only on the interfacial slip velocity $v \equiv \dot{\epsilon}_{x}$, i.e., $f(v)$. We focus on velocity-strengthening interfaces, $f^{\prime}(v)>0$, because in this case sliding is unconditionally stable for symmetric systems [57,75,76]; thus, the origin of any emerging instability must be associated with the absence of geometrical reflection symmetry. Moreover, steady-state velocity-strengthening friction has recently been shown to be a generic feature of dry interfaces over some velocity range [6]. Finally, to simplify the analysis further, we consider the case in which the lower block is much higher than the upper one, $\eta \gg 1$. In other words, we take the limit $H^{(2)} \rightarrow \infty$, such that $H^{(1)} \equiv H$ is the only length scale in the problem.

Under what conditions is homogeneous sliding stable? This question, which is of fundamental importance in a broad range of frictional problems (see, for example, Refs. $[3,20,23,63,75,77-84])$, is first investigated in the context of the simplified problem defined above. As the interface is characterized by velocity-strengthening friction, $f^{\prime}(v)>0$, friction itself tends to stabilize sliding. Consequently, the only possible destabilizing piece of physics is the geometric-asymmetry-induced coupling between interfacial slip and normal stress variations, encapsulated in the function $G_{y}$ [cf. Eq. (3)], which also played a crucial role in Sec. III. Can geometric asymmetry destabilize velocity-strengthening frictional interfaces in much the same way as material asymmetry (the bimaterial effect) can [23]?

To address the stability question, we perturb Eq. (9) to linear order, obtaining [57]

$$
\mu G_{x}(c, k)+i \mu f G_{y}(c, k)+i c \sigma_{0} \delta f / \delta v=0,
$$

which is an implicit equation defining the linear stability spectrum $c(k)$. In the simple velocity-dependent friction case considered here, we have $\delta f / \delta v=f^{\prime}(v)$ (more general interfacial constitutive laws are considered in Sec. IV B). Perturbations with $\operatorname{Im}[c]>0$ are unstable and will grow exponentially, while perturbations with $\operatorname{Im}[c]<0$ are stable (remember that $k>0$ ). An explicit calculation shows that $G_{y}$ reads [57]

$$
G_{y}=\frac{c_{s}^{2}}{c^{2}}\left(\frac{2\left(\alpha_{s}^{2}+1\right)}{1+\tanh \left(k H \alpha_{d}\right)}-\frac{2\left(\alpha_{s}^{2}+1\right)}{1+\tanh \left(k H \alpha_{s}\right)}\right),
$$

where $c_{s}$ and $c_{d}$ are, respectively, the shear and dilatational wave-speeds and $\alpha_{s, d}^{2} \equiv 1-c^{2} / c_{s, d}^{2}$ is introduced.

The limit $H \rightarrow \infty$ amounts to a symmetric system, in which case $\eta \rightarrow 1$, and indeed $G_{y}$ vanishes in this limit. Thus, we can expect the system to be unconditionally stable for $H \rightarrow \infty$. Note that $G_{y}$ also vanishes in the limit $H \rightarrow 0$. Similarly, $G_{x}$ takes the form [57]

$$
G_{x}=\frac{c_{s}^{2}}{c^{2}}\left(\frac{\left(\alpha_{s}^{2}+1\right)^{2} \alpha_{s}^{-1}}{1+\tanh \left(k H \alpha_{s}\right)}-\frac{4 \alpha_{d}}{1+\tanh \left(k H \alpha_{d}\right)}\right) .
$$

Equipped with the results for the dynamic response functions $G_{i}(c, k)$, the implicit equation for the spectrum, Eq. (10), can, in principle, be solved, at least numerically. The equation admits a few solution branches, and in general, its analysis is far from trivial. However, since the purpose of the present discussion is not a complete analysis of Eq. (10) but rather a demonstration of the qualitative effect of the absence of geometrical reflection symmetry, we focus here on a particular branch of solutions, which is shown in Fig. 4(a). It is observed that for a range of parameters, and for a finite range of wave numbers, the solutions are unstable $(\operatorname{Im}[c]>0)$. This is direct numerical evidence that geometric asymmetry can destabilize systems that are otherwise stable [remember that $\left.f^{\prime}(v)>0\right]$.
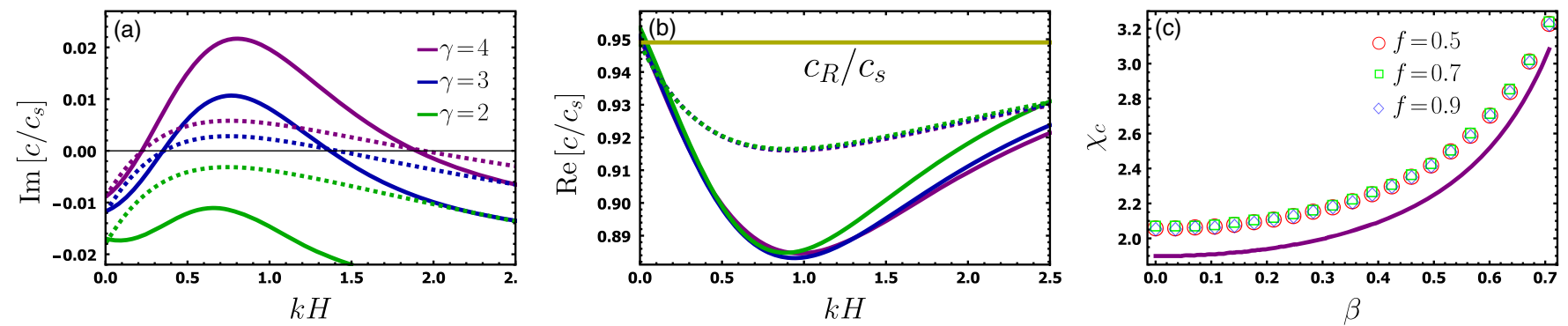

FIG. 4. Linear stability: Simplified analysis. Imaginary (a) and real (b) parts of solutions to the linear stability spectrum in Eq. (10). Here, $\operatorname{Im}(c)>0$ implies an instability. Note that only one solution branch is discussed (other solution branches exist as well, but they are not discussed here). The solid lines show numerical solutions to Eq. (10), and the dashed lines show the approximate analytic solutions obtained by a linear expansion around $c=c_{R}$. The parameters used are $f=0.9$ and $\beta=0.3$, where $\gamma \equiv \mu /\left(\sigma_{0} c_{s} f^{\prime}(v)\right)$ is varied according to the legend. (c) The instability threshold $\chi_{c}$ (i.e., for $\chi \equiv \gamma f<\chi_{c}$, sliding is stable for all $k$ ) vs $\beta \equiv c_{s} / c_{d}$. The open symbols show direct numerical results, and the solid line is the prediction in Eq. (13). 
It seems natural at this point to ask under what conditions this instability is observed. What are the conditions on the various system parameters such that there will be a range of $k$ 's for which $\operatorname{Im}[c(k)]>0$ ? As a prelude, we perform a dimensional analysis. Clearly, the only length scale in the problem is $H$, and indeed the wave number $k$ only appears in the dimensionless combination $k H$. Thus, large (small) $k$ is equivalent to large (small) $H$, and since $G_{y}$ vanishes in both limits $H \rightarrow 0$ and $H \rightarrow \infty$, we expect to find unstable modes only in a finite range $k_{\min }<k<k_{\max }$, if any.

Another dimensionless combination is $\gamma \equiv \mu /\left(\sigma_{0} c_{s} f^{\prime}(v)\right)$, which is the ratio of the elastodynamic quantity $\mu / c_{s}-$ proportional to the so-called radiation damping factor for sliding $[23,26,75,85]$ — and the response of the frictional stress to variations in the sliding velocity. As such, $\gamma$ quantifies the importance of elastodynamics, which tends to destabilize sliding when geometrical asymmetry is present, relative to velocity-strengthening friction, which generically stabilizes sliding. We thus expect large $\gamma$ to promote instability, if $G_{y} \neq 0$. In addition, as $G_{y}$ is the only possible source of instability in the problem, the appearance of $f G_{y}$ is associated with destabilization [because $f$ and $G_{y}$ enter the spectrum in Eq. (10) only through the combination $\left.f G_{y}\right]$. Finally, the ratio of the two wave-speeds $\beta \equiv c_{s} / c_{d}=$ $\sqrt{(1-2 \nu) /(2-2 \nu)}$ is also a dimensionless parameter of the system, which depends only on the bulk Poisson ratio.

To obtain analytic insight into the instability presented in Fig. 4(a), note that solutions in this instability branch are located near the Rayleigh wave-speed, as shown in Fig. 4(b) (note that here $c_{R} \simeq 0.95 c_{s}$ ). Consequently, we expand Eq. (10) to linear order around $c=c_{R}+\delta c$, obtaining an explicit expression for $\delta c(k H)$ [57]. A consequence of this expansion is that the transition between stable and unstable modes occurs for $k$ 's, which approximately satisfy [57]

$$
\gamma f G_{y}\left(c_{R}, k\right) \approx-c_{R} / c_{s} .
$$

This approximate stability criterion explains the existence of an instability and in fact gives reasonable quantitative estimates for its onset.

To see this, note that since $G_{y}\left(c_{R}, k\right)$ [which is negative, cf. Eq. (11) and Ref. [57]) vanishes for both $k=0$ and $k=\infty$, and attains a global minimum for $k$ of order $H^{-1}$, Eq. (13) admits solutions only for certain values of the product $\chi \equiv \gamma f$. When $\chi$ is smaller than a critical value $\chi_{c}$, no solutions exist, and this branch of solutions is stable for all wave numbers. Note that this criterion has exactly the expected structure: The instability is indeed governed by $G_{y}$, and large $\gamma$ or $f$ promotes instability, which only happens at a finite range of wave numbers. These predictions are quantitatively verified in Fig. 4(c). In addition, the real and imaginary parts of the approximate solution for $\delta c(k H)$ [57] are added to Figs. 4(a) and 4(b) (dashed lines), demonstrating reasonable quantitative agreement with the full numerical solution for various parameters.

The results presented in this section demonstrate the destabilizing role that the absence of geometrical reflection symmetry may play in frictional dynamics. In the next section, we significantly extend the analysis to include more realistic friction laws and any geometric contrast.

\section{B. Generalized analysis: State dependence, memory length, and arbitrary geometric asymmetry}

The analysis presented in the previous section adopted two simplifying assumptions, i.e., that the frictional response depends only on the instantaneous slip velocity $v$ and that the lower block is much higher than the upper one, $\eta \rightarrow \infty$. Frictional interfaces, however, are known to also depend on the state of the interface, not just on the slip velocity, and obviously the sliding bodies can feature any geometric asymmetry; i.e., the system can attain any value of $\eta$. Consequently, our goal here is to relax these simplifying assumptions and to present a significantly generalized analysis applicable to a broad range of realistic frictional systems.

It is experimentally well established that the response of frictional interfaces depends, in addition to the slip velocity $v$, on the state of the interface through the (normalized) real contact area $A(\phi) \propto \sigma_{y y}(1+\psi(\phi))$ [1-3], as discussed in relation to Eq. (4). The auxiliary internal state variable $\phi$, which represents the age or maturity of the contact and is of time dimensions, carries memory of the history of the interface. This implies that irrespective of the exact functional form of $\psi(\phi)$ (with $d \psi / d \phi>0$ ), the frictional response $f(\cdot)$ in Eq. (9) depends on both $v$ and $\phi$; i.e., we have $f(v, \phi)$. Since $f$ does not depend solely on the instantaneous sliding velocity, but also on $\phi$, one should distinguish between

$\partial_{v} f \equiv \frac{\partial f(v, \phi)}{\partial v} \quad$ and $\quad d_{v} f \equiv \frac{d f\left(v, \phi_{0}(v)\right)}{d v}$,

where $\phi_{0}(v)$ is the steady-state value of $\phi$.

It is also well established that after a rapid variation in $v$, accompanied by an instantaneous frictional response characterized by $\partial_{v} f$, a new steady state is established over a characteristic slip distance $D$, which can be regarded as an interfacial memory length. This generic behavior is described by the following evolution equation for $\phi$ $[1,3,77]$,

$$
\dot{\phi}=g\left(\frac{v \phi}{D}\right)
$$

with $g(1)=0$ and $g^{\prime}(1)<0$. While several functions $g(\cdot)$ were proposed and extensively studied in the literature $[1,3]$, the only property that affects the linear stability is $g^{\prime}(1)$. Note that if $g(0)>0$ (corresponding to $v=0$ ), the 
equation describes frictional aging ( $\phi$ increases linearly with time under quiescent conditions) and that $g(1)=0$ corresponds to a steady state $\dot{\phi}=0$, implying $\phi_{0}(v)=D / v$. The latter describes contact rejuvenation, where the typical contact lifetime is inversely proportional to $v$.

The physics incorporated in the distinction between $\partial_{v} f$ and $d_{v} f$, and in the memory length $D$-within the so-called rate-and-state friction constitutive framework-implies the existence of two dimensionless parameters that are absent in the simplified analysis of Sec. IVA,

$$
\Delta \equiv \frac{d_{v} f}{\partial_{v} f}, \quad \xi \equiv \frac{D c_{s}}{H v\left|g^{\prime}(1)\right|} .
$$

Frictional interfaces generically feature $\partial_{v} f>0$ [1-3], which is termed the "direct effect" (associated with thermally activated rheology $[3,75])$. As in Sec. IVA, we are interested in $d_{v} f>0$ (i.e., in steady-state velocity-strengthening friction), which implies a positive $\Delta$. In fact, $\Delta$ varies in the range $0<\Delta<1$ [23], while $\xi$ can attain any positive value.

Within this generalized framework, $\delta f / \delta v$ of Eq. (10) takes the form [57]

$$
\frac{\delta f}{\delta v}=\partial_{v} f\left(1+\frac{\Delta-1}{1-i \xi \frac{c}{c_{s}} k H}\right) .
$$

In the limit $\Delta \rightarrow 1$ [i.e., when there is no distinction between $\partial_{v} f$ and $\left.d_{v} f\left(\partial_{v} f \rightarrow d_{v} f\right)\right]$ and when $\xi \rightarrow 0$ (i.e., when the memory length $D$ becomes vanishingly small), we obtain $\delta f / \delta v \rightarrow d_{v} f$. This recovers the result of Sec. IVA, where $d_{v} f$ simply identifies with $f^{\prime}(v)$.

To understand the effect of $\Delta$ and $\xi$ on frictional stability, we need to solve Eq. (10) using Eq. (17). As we also want to consider arbitrary values of the height ratio $\eta$, we should first derive expressions for the interfacial elastodynamic transfer function $G_{x, y}$ for any $\eta$. The generalized result takes the form [57]

$$
\begin{aligned}
G_{x} & =\frac{c_{s}^{2}\left(1+\alpha_{s}^{2}\right)^{2}\left(\tanh \left(\eta k H \alpha_{d}\right)+\tanh \left(k H \alpha_{d}\right)\right)-4 \alpha_{d} \alpha_{s}\left(\tanh \left(\eta k H \alpha_{s}\right)+\tanh \left(k H \alpha_{s}\right)\right)}{c^{2} \alpha_{s}\left(\tanh \left(\eta k H \alpha_{d}\right)+\tanh \left(k H \alpha_{d}\right)\right)\left(\tanh \left(\eta k H \alpha_{s}\right)+\tanh \left(k H \alpha_{s}\right)\right)} \\
G_{y} & =\frac{2 c_{s}^{2}\left(1+\alpha_{s}^{2}\right)}{c^{2}} \frac{\tanh \left(k H \alpha_{s}\right) \tanh \left(\eta k H \alpha_{d}\right)-\tanh \left(k H \alpha_{d}\right) \tanh \left(\eta k H \alpha_{s}\right)}{\left(\tanh \left(\eta k H \alpha_{d}\right)+\tanh \left(k H \alpha_{d}\right)\right)\left(\tanh \left(\eta k H \alpha_{s}\right)+\tanh \left(k H \alpha_{s}\right)\right)} .
\end{aligned}
$$

Note that Eqs. (11) and (12) are obtained from Eq. (18) by taking the $\eta \rightarrow \infty$ limit, which amounts to setting $\tanh \left(\eta k H \alpha_{i}\right)$ to unity (since both $k$ and $\operatorname{Re}\left[\alpha_{i}\right]$ are positive). In addition, as expected, $G_{y}$ vanishes for symmetric systems, i.e., for $\eta=1$.

We are now ready to study the effect of the geometric dimensionless parameter $\eta$, and of the constitutive dimensionless parameters $\Delta$ and $\xi$, on the linear stability of frictional interfaces. In other words, we aim at solving the implicit linear stability spectrum in Eq. (10), with Eqs. (17) and (18). The ultimate goal of such a generalized linear stability analysis is to derive the stability phase diagram in the $\gamma$ [here, $\partial_{v} f$ replaces $f^{\prime}(v)$ in the definition of $\gamma$ in Sec. IVA], $f, \beta, \eta, \Delta$, and $\xi$ parameter space, where the stability boundary is a complex hypersurface in this multidimensional space.

As it is obviously impossible to visualize this highdimensional stability boundary, and in order to gain clear physical insight, we analyze this hypersurface by studying its sections along various parameter directions. A first step was done in Sec. IVA, where the analysis was performed for fixed values of geometric asymmetry $\eta$, frictional resistance $f$, and wave-speed ratio $\beta$, while $\gamma$ varied. As a simple velocity-dependent friction model was adopted there, we also had $\Delta=1$. As observed in Fig. 4(a) and analyzed theoretically in relation to Eq. (13), an instability emerges when $\gamma$ becomes sufficiently large (here, somewhere between $\gamma=2$ and $\gamma=3)$. As $\gamma=\mu /\left(\sigma_{0} c_{s} \partial_{v} f\right)$ quantifies the importance of elastodynamics relative to instantaneous velocity-strengthening friction, the instability emerges when elastodynamics becomes more dominant in the presence of large geometric asymmetry, $\eta=\infty$.

Our next step is to isolate the geometric asymmetry effect embodied in $\eta$. We therefore use the parameters of Fig. 4(a) and 4(b), together with $\gamma=3$, and vary $\eta$ over a very broad range, essentially from $\eta=1$ (corresponding to a symmetric system) to $\eta=\infty$. Note that $\operatorname{Im}\left[c(k H) / c_{s}\right]$, obtained by numerically solving Eqs. (10), (17), and (18), is shown in Fig. 5(a). It is observed that for symmetric systems, $\eta=1$, sliding is stable for all wave numbers. As $\eta$ is increased, $\operatorname{Im}\left[c(k H) / c_{s}\right]$ approaches the $x$ axis until they first intersection when $\eta \simeq 3.3$ at $k H \sim \mathcal{O}(1)$, signaling the onset of instability. This result provides direct evidence for the destabilizing role played by geometric asymmetry in frictional sliding. As $\eta$ is further increased, the system becomes more unstable in the sense of an increased range of unstable wave numbers and a larger growth rate. Obviously, the result in the $\eta=\infty$ limit identifies with that of Fig. 4(a). In fact, the $\eta=\infty$ analysis captures the salient features of the instability spectrum well for $\eta$ values moderately above the critical value $\eta \simeq 3.3$.

Next, we attempt to understand the effect of $\Delta$, i.e., of a difference between the instantaneous response $\partial_{v} f$ and the 

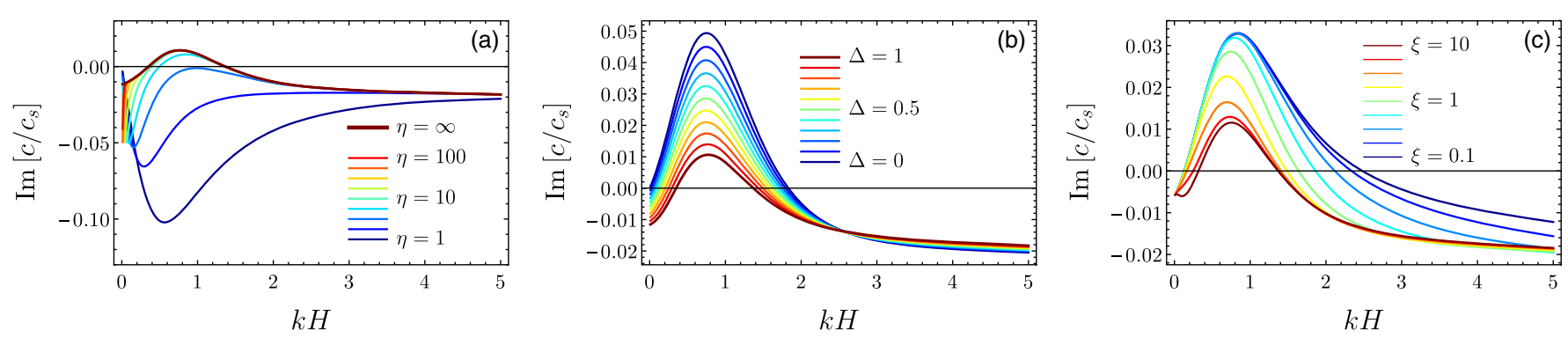

FIG. 5. Linear stability: Generalized analysis. $\operatorname{Im}\left[c / c_{s}\right]$ (i.e., the rate of exponential growth or decay of perturbations; $\operatorname{Im}[c]>0$ corresponds to instability) vs $k H$ for a broad range of physical parameters. In all panels, the parameters are the same as in Fig. 4 with $\gamma=3$. (a) The dependence of $\operatorname{Im}\left[c(k H) / c_{s}\right]$ on $\eta$ for $\Delta=1$. The curve $\eta=\infty$ identifies with the blue curve of Fig. 4(a). The case $\eta=1$ corresponds to a symmetric system and is thus stable for all $k$. (b) The dependence of $\operatorname{Im}\left[c(k H) / c_{s}\right]$ on $\Delta$ for $\xi=1$ and $\eta=\infty$. (c) The dependence of $\operatorname{Im}\left[c(k H) / c_{s}\right]$ on $\xi$ for $\Delta=0.5$ and $\eta=\infty$.

steady-state response $d_{v} f$, on the sliding stability in the presence of geometric asymmetry. For that aim, we plot in Fig. 5(b) $\operatorname{Im}\left[c(k H) / c_{s}\right]$ for various values of $\Delta$, spanning the whole range $0<\Delta<1$, and for fixed $\xi=1$ and $\eta=\infty$. It is observed that as $d_{v} f$ decreases relative to $\partial_{v} f$, i.e., as $\Delta$ decreases, sliding becomes less stable, resulting in a broader range of unstable wave numbers and a larger instability growth rate. This result demonstrates the stabilizing role played by steady-state velocity-strengthening friction in frictional sliding. We note, though, that the qualitative properties of the instability spectrum are rather well captured by the $\Delta=1$ analysis (i.e., for velocity-dependent friction, where no distinction is made between $d_{v} f$ and $\partial_{v} f$ ). We stress that while $\Delta$ affects the properties of instability, the origin of instability is still geometric asymmetry (i.e., sufficiently large $\eta$ ).

Finally, we explore the effect of varying the interfacial memory length $D$, corresponding to varying $\xi$, on frictional stability in the presence of geometric asymmetry. We plot in Fig. 5(c) $\operatorname{Im}\left[c(k H) / c_{s}\right]$ for a broad range of $\xi$ values, and for fixed $\eta=\infty$ and $\Delta=0.5$. It is observed that increasing $D$ (i.e., $\xi$ ) tends to stabilize sliding (i.e., shrink the instability range and growth rate) as it makes the real contact area less sensitive to slip-velocity perturbations. We also stress here that while $\xi$ affects the range and growth rate of instability, its origin is geometric asymmetry (i.e., sufficiently large $\eta$ ).

The results presented in this section provide a rather comprehensive physical picture of the implications of geometric asymmetry on the stability of frictional sliding and of the interplay between geometric asymmetry and generic constitutive properties of frictional interfaces, most notably, the effect of the state of the interface and of an interfacial memory length. The results significantly extend those presented in Sec. IVA, yet they show that the simplified analysis properly captured the destabilizing geometric asymmetry effect. We stress again that additional solutions to Eq. (10) [with Eqs. (17) and (18)] exist. These additional solution branches, along with a more detailed analysis of the multidimensional stability phase diagram, will be presented in a follow-up report.

The results presented in this section regarding the stability of homogeneous sliding in the presence of geometric asymmetry may have far-reaching implications for the dynamics of frictional interfaces in a variety of frictional systems. Under homogeneous loading applied to the top of long-enough sliding bodies, as assumed in the analysis, we predict that no homogeneous steady state will be established experimentally under certain conditions that were carefully quantified. Instead, the interface separating geometrically asymmetric bodies will experience inhomogeneous slip related to the most unstable mode identified in the analysis. This will lead to spatiotemporal stick-sliplike motion, accompanied by distinct acoustic signature as in squeaking door hinges.

In frictional systems where the loading configuration promotes inhomogeneous slip, the results obtained may still be relevant. Inhomogeneous slip in slowly driven frictional interfaces typically takes the form of an expanding creep patch. The conditions under which an expanding creep patch spontaneously generates rapid/unstable slip, an important process known as nucleation, may be related to the minimal unstable wavelength in the stability analysis presented in this section for geometrically asymmetric systems. In particular, the minimal unstable wavelength may determine the size at which the expanding creep patch loses stability.

Finally, when rapid slip develops, it is typically mediated by the propagation of rupture modes. Which mode is actually realized in a given experimental system may be affected by the stability analysis presented here. In particular, extended cracklike rupture modes leave behind a homogeneous sliding state, which may be precluded under certain conditions predicted by our analysis. Instead, localized pulselike rupture modes may develop. Consequently, the results presented in this section may affect rupture-mode selection, a basic open problem in the field of friction. Additional theoretical and experimental 
research should be carried out in order to fully explore these potential implications.

\section{CONCLUDING REMARKS}

In this paper, combining experiments and theory, we showed that frictional interfaces that separate bodies made of identical materials but lack geometric reflection symmetry about the interface generically feature coupling between interfacial slip and normal stress variations. This geometric asymmetry effect is shown to account for a sizable, and previously unexplained, normal-stressinduced weakening effect in frictional cracks. New experiments support the theoretical predictions. We then showed that geometric asymmetry can destabilize homogeneous sliding with velocity-strengthening friction that is otherwise stable. These analyses demonstrate that the effect of geometric asymmetry resembles, sometimes qualitatively and sometimes semiquantitatively, that of material asymmetry (the bimaterial effect).

Since no system is perfectly symmetric, we expect the geometrically induced coupling between interfacial slip and normal stress variations to generically exist in a broad range of man-made and natural frictional systems. Consequently, the coupling should be incorporated into various theoretical approaches and engineering models, as well as employed in interpreting experimental observations. The implications in geophysical contexts, such as in subduction-zone sliding [cf. Fig. 1(c)], call for further investigation.

\section{ACKNOWLEDGMENTS}

E. B. acknowledges support from the Israel Science Foundation (Grant No. 295/16), the William Z. and Eda Bess Novick Young Scientist Fund, COST Action MP1303, and the Harold Perlman Family. J. F. and E. B. acknowledge support from the James S. McDonnell Fund (Grant No. 220020221). J. F. and I. S. acknowledge support from the European Research Council (Grant No. 267256) and the Israel Science Foundation (Grants No. 76/11 and No. 1523/15).

M. A. and Y. B.-S. contributed equally to this work.

[1] C. J. Marone, Laboratory-Derived Friction Laws and Their Application to Seismic Faulting, Annu. Rev. Earth Planet Sci. 26, 643 (1998).

[2] M. Nakatani, Conceptual and Physical Clarification of Rate and State Friction: Frictional Sliding as a Thermally Activated Rheology, J. Geophys. Res. 106, 13347 (2001).

[3] T. Baumberger and C. Caroli, Solid Friction from Stick-Slip Down to Pinning and Aging, Adv. Phys. 55, 279 (2006).

[4] T. Putelat, J. H. Dawes, and J. R. Willis, On the Microphysical Foundations of Rate-and-State Friction, J. Mech. Phys. Solids 59, 1062 (2011).
[5] M. J. Ikari, C. J. Marone, D. M. Saffer, and A. J. Kopf, Slip Weakening as a Mechanism for Slow Earthquakes, Nat. Geosci. 6, 468 (2013).

[6] Y. Bar-Sinai, R. Spatschek, E. A. Brener, and E. Bouchbinder, On the Velocity-Strengthening Behavior of Dry Friction, J. Geophys. Res. Solid Earth 119, 1738 (2014).

[7] G. Di Toro, D. L. Goldsby, and T.E. Tullis, Friction Falls Towards Zero in Quartz Rock as Slip Velocity Approaches Seismic Rates, Nature (London) 427, 436 (2004).

[8] J. R. Rice, Heating and Weakening of Faults During Earthquake Slip, J. Geophys. Res. 111, B05311 (2006).

[9] D. L. Goldsby and T. E. Tullis, Flash Heating Leads to Low Frictional Strength of Crustal Rocks at Earthquake Slip Rates, Science 334, 216 (2011).

[10] J. C. Chang, D. A. Lockner, and Z. Reches, Rapid Acceleration Leads to Rapid Weakening in Earthquake-like Laboratory Experiments, Science 338, 101 (2012).

[11] J. Weertman, Dislocations Moving Uniformly on the Interface Between Isotropic Media of Different Elastic Properties, J. Mech. Phys. Solids 11, 197 (1963).

[12] J. Weertman, Unstable Slippage Across a Fault that Separates Elastic Media of Different Elastic Constants, J. Geophys. Res. 85, 1455 (1980).

[13] L. Freund, Dynamic Fracture Mechanics (Cambridge University Press, Cambridge, England, 1990), p. 563.

[14] G. G. Adams, Self-Excited Oscillations of Two Elastic HalfSpaces Sliding with a Constant Coefficient of Friction, J. Appl. Mech. 62, 867 (1995).

[15] J. Martins and F. M. F. Simões, in 14 Contact Mechanics, edited by M. Raous, M. Jean, and J. Moreau (Springer, New York, 1995), pp. 95-106.

[16] J. A. C. Martins, J. Guimaraes, and L. O. Faria, Dynamic Surface Solutions in Linear Elasticity and Viscoelasticity with Frictional Boundary Conditions, J. Vib. Acoust. 117, 445 (1995).

[17] D. J. Andrews and Y. Ben-Zion, Wrinkle-like Slip Pulse on a Fault Between Different Materials, J. Geophys. Res. Solid Earth 102, 553 (1997).

[18] G. G. Adams, Steady Sliding of Two Elastic Half-Spaces with Friction Reduction due to Interface Stick-Slip, J. Appl. Mech. 65, 470 (1998).

[19] G. G. Adams, An Intersonic Slip Pulse at a Frictional Interface Between Dissimilar Materials, J. Appl. Mech. 68, 81 (2001).

[20] K. Ranjith and J. R. Rice, Slip Dynamics at an Interface Between Dissimilar Materials, J. Mech. Phys. Solids 49, 341 (2001).

[21] M. Adda-Bedia and M. Ben Amar, Self-Sustained Slip Pulses of Finite Size Between Dissimilar Materials, J. Mech. Phys. Solids 51, 1849 (2003).

[22] D. Kammer, V. Yastrebov, G. Anciaux, and J. Molinari, The Existence of a Critical Length Scale in Regularised Friction, J. Mech. Phys. Solids 63, 40 (2014).

[23] E. A. Brener, M. Weikamp, R. Spatschek, Y. Bar-Sinai, and E. Bouchbinder, Dynamic Instabilities of Frictional Sliding at a Bimaterial Interface, J. Mech. Phys. Solids 89, 149 (2016).

[24] R. Madariaga, Dynamics of an Expanding Circular Fault, Bull. Seismol. Soc. Am. 66, 639 (1976). 
[25] R. Madariaga, High-Frequency Radiation from Crack (Stress Drop) Models of Earthquake Faulting, Geophys. J. Int. 51, 625 (1977).

[26] J. R. Rice, Spatio-Temporal Complexity of Slip on a Fault, J. Geophys. Res. 98, 9885 (1993).

[27] Y. Ben-Zion and J. R. Rice, Slip Patterns and Earthquake Populations Along Different Classes of Faults in Elastic Solids, J. Geophys. Res. 100, 12959 (1995).

[28] E. Fukuyama and R. Madariaga, Rupture Dynamics of a Planar Fault in a 3D Elastic Medium: Rate- and SlipWeakening Friction, Bull. Seismol. Soc. Am. 88, 1 (1998).

[29] K. B. Broberg, Cracks and Fracture (Elsevier, New York, 1999).

[30] N. Lapusta, J. R. Rice, Y. Ben-Zion, and G. Zheng, Elastodynamic Analysis for Slow Tectonic Loading with Spontaneous Rupture Episodes on Faults with Rate- and State-Dependent Friction, J. Geophys. Res. 105, 23765 (2000).

[31] Y. Ben-Zion, Dynamic Ruptures in Recent Models of Earthquake Faults, J. Mech. Phys. Solids 49, 2209 (2001).

[32] C. H. Scholz, The Mechanics of Earthquakes and Faulting (Cambridge University Press, Cambridge, England, 2002).

[33] A. M. Rubin and J.-P. Ampuero, Earthquake Nucleation on (Aging) Rate and State Faults, J. Geophys. Res. 110, B11312 (2005).

[34] E. M. Dunham, Dissipative Interface Waves and the Transient Response of a Three-Dimensional Sliding Interface with Coulomb Friction, J. Mech. Phys. Solids 53, 327 (2005).

[35] E. M. Dunham, Conditions Governing the Occurrence of Supershear Ruptures under Slip-Weakening Friction, J. Geophys. Res. 112, B07302 (2007).

[36] J.-P. Ampuero and A. M. Rubin, Earthquake Nucleation on Rate and State Faults-Aging and Slip Laws, J. Geophys. Res. 113, B01302 (2008).

[37] J. W. Rudnicki and J. R. Rice, Effective Normal Stress Alteration due to Pore Pressure Changes Induced by Dynamic Slip Propagation on a Plane Between Dissimilar Materials, J. Geophys. Res. 111, B10308 (2006).

[38] E. M. Dunham and J. R. Rice, Earthquake Slip Between Dissimilar Poroelastic Materials, J. Geophys. Res. 113, B09304 (2008).

[39] M. Comninou, Interface Crack with Friction in the Contact Zone, J. Appl. Mech. 44, 780 (1977).

[40] M. Comninou, The Interface Crack, J. Appl. Mech. 44, 631 (1977).

[41] M. Comninou and D. Schmueser, The Interface Crack in a Combined Tension-Compression and Shear Field, J. Appl. Mech. 46, 345 (1979).

[42] K. Xia, A. J. Rosakis, and H. Kanamori, Laboratory Earthquakes: The Sub-Rayleigh-to-Supershear Rupture Transition, Science 303, 1859 (2004).

[43] J.-P. Ampuero and Y. Ben-Zion, Cracks, Pulses and Macroscopic Asymmetry of Dynamic Rupture on a Bimaterial Interface with Velocity-Weakening Friction, Geophys. J. Int. 173, 674 (2008).

[44] H. Shlomai and J. Fineberg, The Structure of Slip-Pulses and Supershear Ruptures Driving Slip in Bimaterial Friction, Nat. Commun. 7, 11787 (2016).

[45] B. A. Erickson and S. M. Day, Bimaterial Effects in an Earthquake Cycle Model Using Rate-and-State Friction, J. Geophys. Res. 121, 2480 (2016).
[46] S. Rubinstein, G. Cohen, and J. Fineberg, Dynamics of Precursors to Frictional Sliding, Phys. Rev. Lett. 98, 226103 (2007).

[47] O. Ben-David, G. Cohen, and J. Fineberg, The Dynamics of the Onset of Frictional Slip, Science 330, 211 (2010).

[48] O. Ben-David, S. M. Rubinstein, and J. Fineberg, Slip-Stick and the Evolution of Frictional Strength, Nature (London) 463, 76 (2010).

[49] I. Svetlizky and J. Fineberg, Classical Shear Cracks Drive the Onset of Dry Frictional Motion, Nature (London) 509, 205 (2014).

[50] E. Bayart, I. Svetlizky, and J. Fineberg, Slippery but Tough: The Rapid Fracture of Lubricated Frictional Interfaces, Phys. Rev. Lett. 116, 194301 (2016).

[51] G. Plafker, Tectonic Deformation Associated with the 1964 Alaska Earthquake: The Earthquake of 27 March 1964 Resulted in Observable Crustal Deformation of Unprecedented Areal Extent, Science 148, 1675 (1965).

[52] S.E. Barrientos and S. N. Ward, The 1960 Chile Earthquake: Inversion for Slip Distribution from Surface Deformation, Geophys. J. Int. 103, 589 (1990).

[53] B. Delouis, J.-M. Nocquet, and M. Vallée, Slip Distribution of the February 27, $2010 \mathrm{Mw}=8.8$ Maule Earthquake, Central Chile, from Static and High-Rate GPS, InSAR, and Broadband Teleseismic Data, Geophys. Res. Lett. 37, L17305 (2010).

[54] R. Madariaga, M. Metois, C. Vigny, and J. Campos, Central Chile Finally Breaks, Science 328, 181 (2010).

[55] M. Simons, S. E. Minson, A. Sladen, F. Ortega, J. Jiang, S. E. Owen, L. Meng, J.-P. Ampuero, S. Wei, R. Chu, D. V. Helmberger, H. Kanamori, E. Hetland, A. W. Moore, and F. H. Webb, The 2011 Magnitude 9.0 Tohoku-Oki Earthquake: Mosaicking the Megathrust from Seconds to Centuries, Science 332, 1421 (2011).

[56] S. León-Ríos, S. Ruiz, A. Maksymowicz, F. Leyton, A. Fuenzalida, and R. Madariaga, Diversity of the 2014 Iquique's Foreshocks and Aftershocks: Clues about the Complex Rupture Process of a Mw 8.1 Earthquake, J. Seismol. 1911, 1 (2016).

[57] See Supplemental Material at http://link.aps.org/ supplemental/10.1103/PhysRevX.6.041023 for additional technical details.

[58] P. Geubelle, A Spectral Method for Three-Dimensional Elastodynamic Fracture Problems, J. Mech. Phys. Solids 43, 1791 (1995).

[59] F. Bowden and D. Tabor, The Friction and Lubrication of Solids (Clarendon Press, Oxford, 1950).

[60] J. H. Dieterich and B. D. Kilgore, Direct Observation of Frictional Contacts: New Insights for State-Dependent Properties, Pure Appl. Geophys. 143, 283 (1994).

[61] M. Nakatani and C. H. Scholz, Intrinsic and Apparent Short-Time Limits for Fault Healing: Theory, Observations, and Implications for Velocity-Dependent Friction, J. Geophys. Res. 111, B12208 (2006).

[62] K. Nagata, M. Nakatani, and S. Yoshida, Monitoring Frictional Strength with Acoustic Wave Transmission, Geophys. Res. Lett. 35, L06310 (2008).

[63] Y. Bar-Sinai, R. Spatschek, E. A. Brener, and E. Bouchbinder, Instabilities at Frictional Interfaces: Creep Patches, Nucleation, and Rupture Fronts, Phys. Rev. E 88, 060403(R) (2013). 
[64] J.H. Dieterich, Time-Dependent Friction in Rocks, J. Geophys. Res. 77, 3690 (1972).

[65] J. H. Dieterich, Time-Dependent Friction and the Mechanics of Stick-Slip, Pure Appl. Geophys. 116, 790 (1978).

[66] A. C. Palmer and J. R. Rice, The Growth of Slip Surfaces in the Progressive Failure of Over-Consolidated Clay, Proc. R. Soc. A Math. Phys. Eng. Sci. 332, 527 (1973).

[67] A. N. B. Poliakov, R. Dmowska, and J. R. Rice, Dynamic Shear Rupture Interactions with Fault Bends and Off-Axis Secondary Faulting, J. Geophys. Res. Solid Earth 107, ESE 6 (2002).

[68] O. Samudrala, Y. Huang, and A. J. Rosakis, Subsonic and Intersonic Shear Rupture of Weak Planes with a Velocity Weakening Cohesive Zone, J. Geophys. Res. 107, 2170 (2002).

[69] L. D. Landau and E. M. Lifshitz, Theory of Elasticity, 3rd ed. (Pergamon Press, New York, 1986), Vol. 7.

[70] B. Read and J. Duncan, Measurement of Dynamic Properties of Polymeric Glasses for Different Modes of Deformation, Polymer Testing 2, 135 (1981).

[71] S. Timoshenko and J. N. Goodier, Theory of Elasticity (McGraw-Hill, New York, 1951).

[72] Note that at large $k W, \mu^{\text {eff }}$ becomes minutely smaller than $\mu$; see Ref. [57].

[73] M. Radiguet, D. Kammer, and J. Molinari, The Role of Viscoelasticity on Heterogeneous Stress Fields at Frictional Interfaces, Mech. Mater. 80, 276 (2015).

[74] D. S. Kammer, M. Radiguet, J.-P. Ampuero, and J.-F. Molinari, Linear Elastic Fracture Mechanics Predicts the Propagation Distance of Frictional Slip, Tribol. Lett. 57, 23 (2015).

[75] J. R. Rice, N. Lapusta, and K. Ranjith, Rate and State Dependent Friction and the Stability of Sliding Between
Elastically Deformable Solids, J. Mech. Phys. Solids 49, 1865 (2001).

[76] H. Perfettini and J.-P. Ampuero, Dynamics of a Velocity Strengthening Fault Region: Implications for Slow Earthquakes and Postseismic Slip, J. Geophys. Res. 113, B09411 (2008).

[77] J. R. Rice and A. Ruina, Stability of Steady Frictional Slipping, J. Appl. Mech. 50, 343 (1983).

[78] B. Armstrong-Hélouvry, P. Dupont, and C. C. De Wit, A Survey of Models, Analysis Tools and Compensation Methods for the Control of Machines with Friction, Automatica 30, 1083 (1994).

[79] H. Olsson, K. Åström, C. C. De Wit, M. Gäfvert, and P. Lischinsky, Friction Models and Friction Compensation, Eur. J. Control 4, 176 (1998).

[80] Q. S. Nguyen, Instability and Friction, C.R. Mec. 331, 99 (2003).

[81] M. J. Ikari, C. J. Marone, and D. M. Saffer, On the Relation Between Fault Strength and Frictional Stability, Geology 39, 83 (2010).

[82] Y. Pomeau and M. Le Berre, Critical Speed-up vs Critical Slow-Down: A New Kind of Relaxation Oscillation with Application to Stick-Slip Phenomena, arXiv:1107.3331.

[83] T. Putelat and J. H. Dawes, Steady and Transient Sliding under Rate-and-State Friction, J. Mech. Phys. Solids 78, 70 (2015).

[84] Y. Bar Sinai, E. A. Brener, and E. Bouchbinder, Slow Rupture of Frictional Interfaces, Geophys. Res. Lett. 39, L03308 (2012).

[85] P. Crupi and A. Bizzarri, The Role of Radiation Damping in the Modeling of Repeated Earthquake Events, Ann. Geophys. 56, R0111 (2013). 